\title{
Serial transthoracic coronary Doppler shows complete reversibility of microvascular obstruction pattern at one month after reperfused acute myocardial infarction.
}

\author{
Camelia Diana Ober ${ }^{1}$, Mihai Claudiu Ober ${ }^{2}$, Adrian Corneliu Iancu ${ }^{1,3}$
}

1"Niculae Stăncioiu" Heart Institute, ${ }^{2}$ Cluj County Emergency Clinical Hospital, 3 "Iuliu Haţieganu" University of Medicine and Pharmacy, Cluj-Napoca, Romania

\begin{abstract}
"No reflow" phenomenon is a common complication with significant prognostic repercussions after reperfused acute myocardial infarction. Previous studies have shown the feasibility and prognostic significance of coronary microcirculation assessment by transthoracic Doppler of left anterior descending artery (LAD). The aim of the study was to evaluate the time course of the coronary microcirculation status after acute anterior myocardial infarction reperfused by primary angioplasty with stent on LAD. Material and methods: Twenty-three patients underwent transthoracic echocardiography at 3 days (M0) and one month (M1) after reperfusion, with LAD flow analysis (peak systolic and diastolic velocities, diastolic deceleration time and systolic flow reversal). The patients were divided into two groups, of good reperfusion and "no reflow", by a $600 \mathrm{~ms}$ cut-off of the M0 diastolic deceleration time, previously shown to best discriminate between the two microvascular reperfusion states. Results: Of all patients, 21 survived to M1 and were included in analysis. Microvascular obstruction, as defined, was present in $50 \%$ of patients at M0 and it was associated with significantly worse ejection fraction both at M0 and M1 ( $\mathrm{p}=0.01$ and 0.04 , respectively). Killip class at admission was the only independent predictor of "no reflow". DDT increased from $661.9 \pm 339.3$ at M0 to $948.2 \pm 201.8$ at M1 ( $p=0.0003)$. In patients with initial "no reflow", DDT increased to normal values at M1 (876.2 $\pm 167.7 \mathrm{vs}$. $346.3 \pm 133.9$ at $\mathrm{M} 0, \mathrm{p}<0.0001)$. In these patients, the PDV/PSV ratio decreased at one month $(1.91 \pm 0.37 \mathrm{vs.} 2.70 \pm 1.22$, $\mathrm{p}=0.06)$, by decrease of PDV $(0.29 \pm 0.06$ vs. $0.40 \pm 0.19, \mathrm{p}=0.03)$. Conclusions: The parameters of coronary microcirculatory impedance improve significantly at one month after infarction, suggesting delayed full reversibility of the microvascular obstruction.

Keywords: "no reflow" reversibility; microvascular obstruction reversibility; coronary Doppler flow; acute myocardial infarction; primary angioplasty
\end{abstract}

\section{Introduction}

"No reflow" phenomenon is a frequent complication of acute myocardial infarction (AMI) reperfused either by pharmacological or by interventional means. It consists of persistent microvascular obstruction, despite permeable epicardial coronary artery, leading to further necrosis in the infarct territory [1-2].

Received 30.06.2016 Accepted 28.08.2016

Med Ultrason

2017, Vol. 19, No 1, 45-50

Corresponding author: Mihai Claudiu Ober Angiography Laboratory

Cluj County Emergency Clinical Hospital

3-5 Clinicilor street,

400006 Cluj-Napoca, Romania

Phone: +40 722325302

E-mail: mihai_ober@yahoo.com
Previous studies showed that "no reflow" phenomenon is present in about half of the patients with AMI reperfused by primary percutaneous intervention (pPCI), and it has prognostic significance, by a larger extent of necrosis, maladaptive left ventricular remodelling, and, consequently, higher incidence of mechanical complications and clinical events [3-5].

There are multiple mechanisms [2,6] involved in the genesis of this phenomenon, but the final, common pathway of these is the microvascular obstruction (MVO), with consequent increase of the microcirculatory impedance. This can be assessed either by intracoronary flow wire $[7,8]$ or by transthoracic Doppler examination [9-11] of coronary arteries, especially of the left anterior descending artery (LAD), which is the most accessible. Previous studies showed the feasibility and the prognostic utility of transthoracic Doppler of LAD in the setting of reperfused AMI [9-11]. 
Various Doppler parameters were proven to correlate with "no reflow" phenomenon [7-11], predicting the lack of myocardial recovery after reperfusion. The increase in microvascular impedance secondary to microcirculatory obstruction is associated with a specific Doppler pattern, which includes an increase of the deceleration slope of the coronary diastolic flow, with consequent shortening of the diastolic deceleration time (DDT) [7-9], increase of the peak diastolic velocity (PDV), and decrease of peak systolic velocity (PSV), up to a systolic flow reversal (SFR) [10], leading to an increase of the ratio of peak diastolic over peak systolic velocities (PDV/PSV) [11].

The most utilised of these parameters is DDT, with a very well validated cut-off of $600 \mathrm{~ms}$ [7-9, 12-15], significant for MVO with prognostic relevance. However, as shown by Okamura et al [12], "no reflow" is not an "all or nothing" phenomenon and there are markers of more severe MVO, as the presence of SFR [12] or shorter DDT, with a threshold of $190 \mathrm{~ms}$ that seems to predict totally non-viable myocardium $[16,17]$.

However, there are less data concerning the time course of the MVO after the acute phase of the infarction, thus the aim of the present study was to assess the time course of Doppler parameters of microvascular resistance at one month, in patients with a first anterior AMI, reperfused by pPCI with stent.

\section{Material and methods}

This is a prospective, observational, longitudinal, single blinded study, which included 23 consecutive patients with a first anterior AMI, reperfused by pPCI with stent on the LAD, admitted at the Heart Institute ClujNapoca, between April and September 2010. The study was approved by the local Ethics Committee. All patients signed an informed consent before the index pPCI. This was an exploratory research, conducted before the randomised phase of our previously published work [18], following the same examination protocols. The diagnosis and treatment of STEMI were made according to the current practice guidelines at the time of the study [19], as decided by the attending physician. All patients were pre-treated with aspirin $325 \mathrm{mg}$ and clopidogrel $600 \mathrm{mg}$, orally, at the first hospital contact. In all patients, unfractioned heparin 100ui/kg was given intravenously in the catheterisation laboratory, before pPCI.

Electrocardiogram was analysed by the attending physician, independent of the present study, at admission and at 90 minutes post $\mathrm{pPCI}$, in order to estimate the STsegment resolution (STR); STR $>70 \%$ in the worst initial lead was taken into account.
Blood samples were obtained on admission; of these, glycaemia ( $\mathrm{mg} / \mathrm{dl})$, leukocyte count, and creatinine clearance $(\mathrm{ml} / \mathrm{min})$ were evaluated as potential predictors of MVO. Serial blood samples were also drawn in order to get the peak of creatin-kinase-MB isoform (CK-MB, $\mathrm{U} / \mathrm{L}$ ), as an estimation of the extent of necrosis.

Coronary angiography after pPCI was analysed by the operator, independent of the present study, by visual estimation, with respect to TIMI flow grading and myocardial blush grading (MBG), as described by TIMI study group [20] and van't Hof et al [21], respectively, in order to estimate perfusion at myocardial level.

Echocardiography was performed at three days (M0) and one month (M1) post-procedure, using a GE Vivid 7 ultrasound system (General Electric, Milwaukee, WI, USA). Standard echocardiographic parameters were recorded, including those regarding left ventricle function as an ejection fraction (EF), end-diastolic volume (EDV), and end-systolic volume (ESV), measured by biplane Simpson's method. Since diastolic function might influence coronary flow, trans-mitral flow and mitral annulus tissue Doppler were analysed as predictors of MVO. Annular E' wave was measured at septal and anterior points of the annulus, and an average of these values was recorded, as a marker of regional diastolic function in the infarct territory. Ratio of mitral E wave velocity and average E' velocity was also calculated, as an estimate of global diastolic function. In addition, the flow velocity profile in the mid-distal LAD was recorded by transthoracic Doppler echocardiography, as previously described [22], in order to determine the parameters associated with MVO, by an experienced operator (C.D.O.), blinded to the angiographic and clinical data. Briefly, in order to record the LAD flow, the transducer was set on the midclavicular line, in an intermediate position between parasternal long axis and apical two-chamber view, and the LAD colour Doppler signal was searched by slight rotation and angulation of the transducer, using the built-in coronary examination preset. After locating the artery, the spectral curve of pulsed-wave Doppler was recorded and analysed. The pulsed-wave Doppler gate length was set to 1 to $3 \mathrm{~mm}$, according to the estimated vessel size.

Consequently, the LAD Doppler flow pattern was recorded, taking into account the parameters correlated with MVO: DDT, PDV, PSV, PDV/PSV, and early SFR. DDT was measured as below shown, on the slope of deceleration of coronary flow, from the diastolic peak to the intersection with the horizontal axis. All parameters were measured on three to five cardiac cycles with the best definition of the velocity curve, and the average of the measurements was recorded. 
The patients were divided by the result of DDT at M0, with a cut-off value of $600 \mathrm{~ms}$, into a "no reflow" Group (NR, DDT $<600 \mathrm{~ms}$ ) and a 'Good Reperfusion' Group (GR, DDT $>600 \mathrm{~ms}$ ). Figure 1 shows the method of measurement of DDT, with typical patterns of good reperfusion (fig 1a) and "no reflow" (fig 1b).

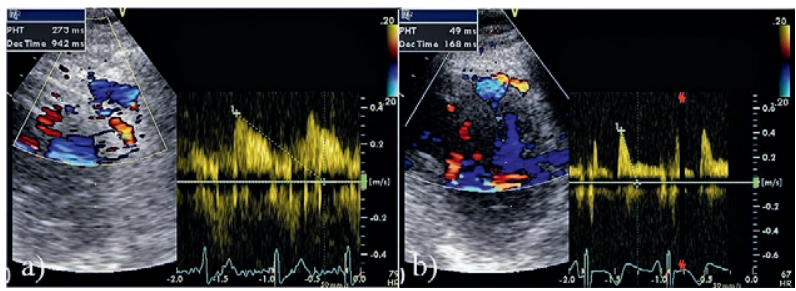

Fig 1. Method of measurement of diastolic deceleration time (DDT), with typical patterns of normal flow (a) and "no reflow" (b).

\section{Statistical analysis}

Statistical analysis was performed in Microsoft Excel 2003 and Epi Info 6.04 (Center for Disease Control, Atlanta, USA). A p value $<0.05$ was interpreted as significant. Continuous variables were expressed as mean \pm standard deviation and they were compared using Student's t test (paired for the comparisons M1 vs. M0, unpaired for other comparisons). Discrete variables are expressed as number (percentage) and they were compared using the $\chi^{2}$ test or Fischer exact test, as appropriate; univariate analysis results are expressed as a relative risk (RR) with Taylor series 95\% confidence limits. Similarly, known markers of myocardial perfusion (TIMI flow, MBG, and STR) were analysed as predictors of the tissue perfusion status. Non-binary variables (Killip class and $\mathrm{MBG}$ ) were compared by $\chi^{2}$ test with the appropriate degrees of liberty.

Table I. Baseline features both for all patients and divided by reperfusion status (univariate predictors of microvascular obstruction).

\begin{tabular}{|c|c|c|c|c|c|}
\hline & $\begin{array}{l}\text { All } \\
(n=23)\end{array}$ & $\begin{array}{l}\text { NR Group } \\
(\mathrm{n}=11)\end{array}$ & $\begin{array}{l}\text { GR Group } \\
(\mathrm{n}=11)\end{array}$ & $\mathbf{p}$ & $\operatorname{RR}(95 \% \mathrm{CI})$ \\
\hline Age (years) & $59.7 \pm 13.2$ & $61.5 \pm 14.2$ & $57.0 \pm 11.9$ & 0.43 & \\
\hline Gender (female) & $8(34.8 \%)$ & $3(27.3 \%)$ & $4(36.4 \%)$ & 1 & $0.80(0.30-2.14)$ \\
\hline Smoking & $12(52.2 \%)$ & $4(36.4 \%)$ & $8(72.7 \%)$ & 0.09 & $0.48(0.19-1.17)$ \\
\hline Hypertension & $14(60.9 \%)$ & $7(63.6 \%)$ & $6(54.5 \%)$ & 1 & $1.21(0.50-2.94)$ \\
\hline Diabetes Mellitus & $6(26.1 \%)$ & $2(18.2 \%)$ & $3(27.3 \%)$ & 1 & $0.76(0.24-2.42)$ \\
\hline Previous Angina & $11(47.8 \%)$ & $5(45.5 \%)$ & $5(45.5 \%)$ & 1 & $1.00(0.43-2.31)$ \\
\hline Killip Class $>1$ & $8(34.7 \%)$ & $7(63.6 \%)$ & $1(9.1 \%)$ & 0.024 & $3.06(1.28-7.30)$ \\
\hline Killip 1 & 15 & 4 & 10 & $0.02 *$ & \\
\hline Killip 2 & 6 & 5 & 1 & & \\
\hline Killip 3 & 2 & 2 & 0 & & \\
\hline TIMI flow pre PCI $>1$ & $9(39.1 \%)$ & $3(27.3 \%)$ & $6(54.5 \%)$ & 0.38 & $0.54(0.20-1.50)$ \\
\hline TIMI 2 & 9 & 3 & 6 & & \\
\hline TIMI 0 & 14 & 8 & 5 & & \\
\hline Pre-dilatation & $7(30.4 \%)$ & $5(45.5 \%)$ & $2(18.2 \%)$ & 0.36 & $1.79(0.82-3.88)$ \\
\hline Body mass index & $27.0 \pm 3.9$ & $28.8 \pm 4.5$ & $26.0 \pm 2.7$ & 0.09 & \\
\hline Leukocyte count (x103/mm3) & $13.0 \pm 4.8$ & $13.5 \pm 4.3$ & $11.6 \pm 5.1$ & 0.35 & \\
\hline Glycaemia (mg/dl) & $158 \pm 79.1$ & $144.3 \pm 48.2$ & $145.7 \pm 53.3$ & 0.95 & \\
\hline Creatinine clearance $(\mathrm{ml} / \mathrm{min})$ & $87.0 \pm 29.9$ & $86.4 \pm 29.2$ & $90.3 \pm 32.7$ & 0.76 & \\
\hline Peak CK-MB (U/L) & $215.0 \pm 143.0$ & $264.6 \pm 116.4$ & $179.2 \pm 159.0$ & 0.17 & \\
\hline Total ischemic time (min) & $352 \pm 181.5$ & $369.1 \pm 195.4$ & $323.6 \pm 177.3$ & 0.57 & \\
\hline Stent length (mm) & $20.8 \pm 3.9$ & $20.9 \pm 3.8$ & $20.5 \pm 4.4$ & 0.80 & \\
\hline Stent diameter $(\mathrm{mm})$ & $3.04 \pm 0.2$ & $3.1 \pm 0.2$ & $3.0 \pm 0.2$ & 0.09 & \\
\hline E'velocity (m/s) & $0.059 \pm 0.018$ & $0.050 \pm 0.015$ & $0.067 \pm 0.017$ & 0.028 & \\
\hline $\mathrm{E} / \mathrm{E}^{\prime}$ ratio & $14.2 \pm 5.8$ & $15.9 \pm 6.9$ & $12.5 \pm 6.9$ & 0.17 & \\
\hline \multicolumn{6}{|l|}{ TIMI flow } \\
\hline TIMI 3 & $20(86.9 \%)$ & $8(72.7 \%)$ & $11(100 \%)$ & 0.21 & $0.42(0.25-0.71)$ \\
\hline TIMI 2 & 3 & 3 & 0 & & \\
\hline MBG (3 vs. < 3) & $4(17.4 \%)$ & $1(9.1 \%)$ & $3(27.3 \%)$ & 0.59 & $0.45(0.08-2.58)$ \\
\hline MBG 0 & 6 & 4 & 2 & $0.57 *$ & \\
\hline MBG 1 & 7 & 4 & 3 & & \\
\hline MBG 2 & 6 & 2 & 3 & & \\
\hline MBG 3 & 4 & 1 & 3 & & \\
\hline $\mathrm{STR}>70 \%$ & $7(30.4 \%)$ & $1(9.1 \%)$ & $6(54.5 \%)$ & 0.06 & $0.21(0.03-1.36)$ \\
\hline
\end{tabular}

$\mathrm{RR}$ - relative risk, CI - Taylor series $95 \%$ confidence interval; ${ }^{*} \chi^{2}$ test with 2 and 3 degrees of liberty, respectively; TIMI - "Thrombolysis in Myocardial Infarction", MBG - myocardial blush grade, STR - ST segment resolution 
The variables with $p<0.1$ at univariate analysis were tested in a stepwise multivariate logistic regression analysis model to identify independent predictors for "no reflow". In addition, the markers of myocardial reperfusion (TIMI 3 flow, MBG 3, and STR) were analysed in a similar stepwise multivariate logistic model as predictors for "no reflow". The results of the multivariate analysis are expressed as odds ratio (OR) with $95 \%$ confidence interval (95\% CI), coefficients (b), standard error (SE), for the variables included in the model, and the $\mathrm{chi}^{2}$ and $\mathrm{p}$ values for the overall model. Logistic regression analysis was performed using Medcalc Software (www.medcalc. org, Ostend, Belgium).

\section{Results}

Of the 23 patients who entered the study, one patient died before M0 examination and other (in the NR group) before M1 (mortality at one month of $8.70 \%$ ), therefore they were excluded from the longitudinal analysis.

Table I shows the demographic data and the baseline features, both for all patients, and divided by DDT at M0, as univariate predictors of microvascular obstruction.

LAD flow was analysable in all patients at both examinations. MVO was present in $11(50 \%)$ of the 22 patients with reperfused anterior AMI, who underwent the M0 echocardiography. Two patients $(9.1 \%)$ showed markers of severe MVO; one patient had DDT $<190 \mathrm{~ms}$ and SFR, and another had SFR only. No significant difference of peak CK-MB was noted between the two groups. Of the analysed variables, only lower E' wave velocity and higher Killip class at admission $(\mathrm{p}=0.028$ and 0.024 , respectively) were significant predictors for "no reflow". Higher body mass index, larger stent diameter, and nonsmoking status showed also a trend to predict MVO, that did not reach statistical significance ( $\mathrm{p}=0.09$, each). These five variables were tested in a stepwise multivariate logistic regression analysis model. Only Killip class $>1$ at admission remained an independent predictor for MVO, $\mathrm{OR}=17.5$ (95\% CI:1.6-191.9), $\mathrm{b}=2.86, \mathrm{SE}=1.22, \mathrm{p}=0.02$, with an overall model fit $\mathrm{chi}^{2}=7.72$ and $\mathrm{p}=0.0055$.

There was no significant correlation between angiographic markers of MVO (TIMI flow, MBG) and DDT group, but an almost significant correlation with ST-segment resolution was noticed $(\mathrm{p}=0.06)$. At the multivariate logistic regression analysis, STR appeared as the only significant independent predictor for $\mathrm{MVO}, \mathrm{OR}=0.08$ (95\% CI:0.0078-0.8947), b=-2.48, SE $=1.21, \mathrm{p}=0.04$, with an overall model fit $\mathrm{chi}^{2}=5.7$ and $\mathrm{p}=0.017$.

The whole group analysis at one month (Table II) showed a significant increase of the left ventricular systolic function, expressed by EF, especially by left ventricle dilatation (significant increase of EDV, without ESV change).

DDT increased significantly at one month, reaching the 'good reperfusion' threshold of $600 \mathrm{~ms}$ in all patients (fig 2a). The PDV/PSV ratio decreased significantly, by a significant decrease of PDV; PSV did not change at M1 vs. M0.

The sub-group analysis (Table III) revealed a significantly greater $\mathrm{EF}$ in the GR group, both at M0 and M1. There was a trend towards increased EF in both groups at M1 vs. M0, significant for the NR group, but non-significant for the GR group. This was based on a significant

Table II. Echocardiographic parameters for the patients followed to M1 (n=21); paired comparison between M0 and M1.

\begin{tabular}{llll}
\hline & M0 & M1 & p \\
\hline EF (\%) & $50.0 \pm 11.1$ & $55.1 \pm 11.3$ & $\mathbf{0 . 0 0 7}$ \\
EDV (ml) & $98.2 \pm 29.2$ & $106.4 \pm 33.2$ & $\mathbf{0 . 0 3}$ \\
ESV (ml) & $48.9 \pm 18.2$ & $48.5 \pm 21.4$ & 0.87 \\
DDT (ms) & $661.9 \pm 339.3$ & $948.2 \pm 201.8$ & $\mathbf{0 . 0 0 0 3}$ \\
PDV/PSV & $2.25 \pm 0.97$ & $1.83 \pm 0.32$ & $\mathbf{0 . 0 2}$ \\
PDV (m/s) & $0.35 \pm 0.16$ & $0.28 \pm 0.06$ & $\mathbf{0 . 0 3}$ \\
PSV (m/s) & $0.16 \pm 0.07$ & $0.16 \pm 0.03$ & 0.67 \\
\hline
\end{tabular}

EF - ejection fraction, EDV - end systolic volume, ESV - end systolic volume, DDT - diastolic deceleration time, PDV - peak diastolic velocity, PSV - peak systolic velocity

Table III. Echocardiographic parameters by the initial status of reperfusion; paired comparison intra-group between M1 and M0 and unpaired comparison between groups at M0 and M1, respectively.

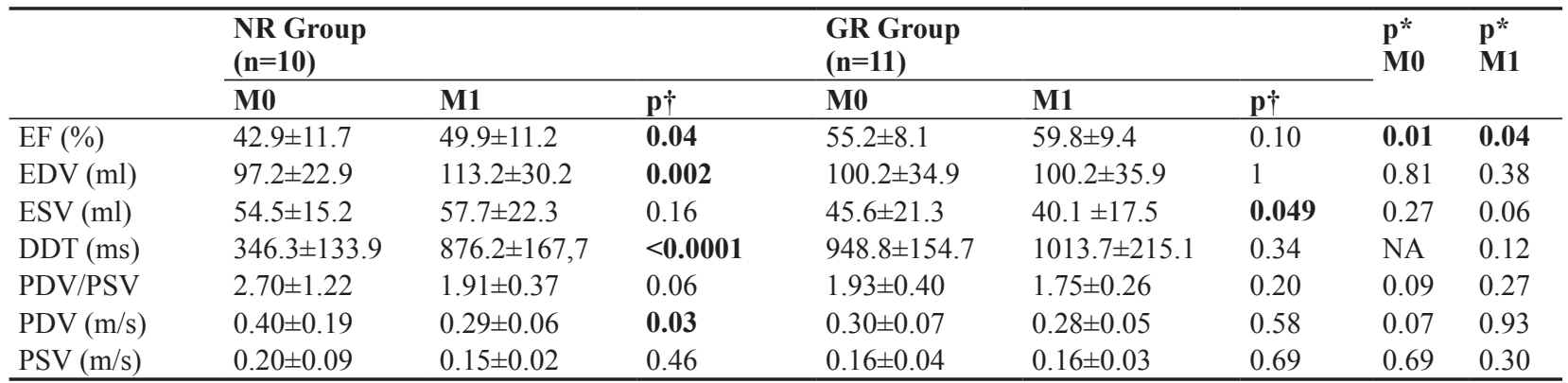

$\dagger$ intra-group, M0 vs. M1; * between groups; NA - not applicable, EF - ejection fraction, EDV - end systolic volume, ESV - end systolic volume, DDT - diastolic deceleration time, PDV - peak diastolic velocity, PSV - peak systolic velocity 


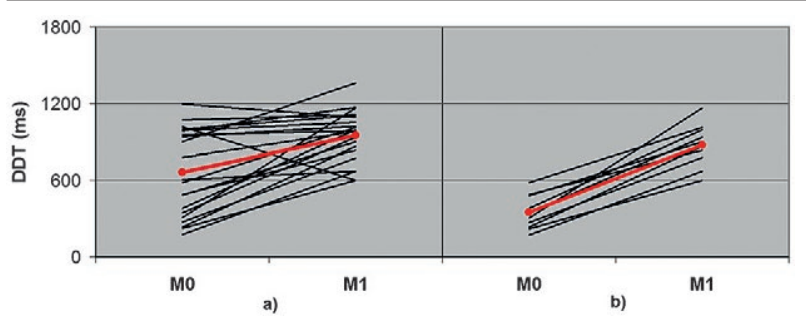

Fig 2. Evolution of DDT from M0 to M1, for the whole population (a) and for the "no reflow" group (b); in red, average evolution, respectively.

increase of EDV in the NR group ( $\mathrm{p}=0.002)$, but on a significant decrease of ESV in GR group ( $\mathrm{p}=0.049)$, leading to a divergent change of the ESV between the two groups, with a trend to smaller ESV at M1 in GR group $(p=0.06)$.

In addition, we noted a highly significant increase of DDT at M1 and a decrease of PDV and PDV/PSV ratio in the NR group (fig 2b), with no significant change in the GR group, leading to "normal" microcirculatory resistance parameters in all patients (including those with severe MVO), with no difference between groups at one month. Of note, SFR was absent in all patients at M1.

\section{Discussions}

Our study shows that the "no reflow" phenomenon, as assessed by transthoracic coronary Doppler echocardiography, is a frequent occurrence after reperfused anterior AMI and it is associated with worse functional recovery. In addition, serial recordings at one month show significant improvement of the coronary Doppler parameters, with complete "normalisation" in the "no reflow" group.

The only independent predictor of microvascular obstruction was Killip class at admission, but the cause-effect relationship is unclear. A possible explanation might be that MVO would be present before reperfusion and it would be associated with worse ventricular function (especially diastolic, as suggested by the lower E' velocity in the MVO group).

Another important finding of our study was the lack of correlation between angiographic markers of MVO (TIMI flow, MBG) and the actual diagnosis of "no reflow" by coronary Doppler; this makes the diagnostic of MVO difficult for the operator in the catheterisation laboratory and it precludes timely intervention against it. Better correlation was shown with ST-segment resolution at 90 minutes, which might be of more help in diagnosis, but usually its assessment is delayed until the exit of the catheterisation laboratory.

There are a few studies showing delayed improvement of microcirculation after "no reflow" phenomenon by coronary Doppler [14,23], cardiac magnetic resonance
$[24,25]$ or contrast echocardiography [26]. However, to the best of our knowledge, this is the first study to show such complete reversal of the Doppler pattern of the MVO associated with "no reflow" phenomenon, at one month.

The proof of reversibility of MVO, even delayed, would be of great conceptual importance, as it would be an incentive to search new means and to use current ones $[1,2,5,6]$ to accelerate this reversal in the acute phase, in order to maximise myocardial salvage. Therefore, given that MVO is a very frequent occurrence and it seems poorly correlated with angiographic markers, it is important to keep in mind the possibility of "no reflow" in all patients with reperfused AMI and to make every effort to revert it as soon as possible with current therapies. However, the fact that MVO might be present before reperfusion could suggest that "no reflow" phenomenon is not a cause, but an effect of more extensive myocardial damage, and it could explain the current relative lack of success of myocardial salvage by MVO-targeted means.

In addition, there could be a practical impact of the demonstration MVO reversibility, as some therapies (e.g. cell therapy) require a permeable microcirculation in order to be effective.

Inherently, there are some limitations in our study. Firstly, the small number of patients; however, the accuracy is increased by the longitudinal design, with self-controlled comparisons. Secondly, the coronary Doppler pattern is a surrogate marker of MVO, but, as shown above, it is well correlated with other methods of diagnosis and it has a prognostic value. Nevertheless, we cannot exclude other mechanisms of decrease of coronary impedance (e.g. collateral development with shunting of microcirculation). Thirdly, because of the difficulty of examination of other arteries, we took into account only anterior infarcts, with LAD lesions; however, the LAD occlusion has the most important prognostic impact and the results may be probably generalised at the concept level.

In conclusion, transthoracic Doppler parameters indicating microvascular obstruction after "no reflow" phenomenon improve significantly at one month, reaching values similar to those recorded in patients with good reperfusion.

\section{Conflict of interest: none}

\section{References}

1. Durante A, Camici PG. Novel insights into an "old" phenomenon: the no reflow. Int J Cardiol 2015;187:273-280.

2. Kaul S. The "no reflow" phenomenon following acute myocardial infarction: mechanisms and treatment options. J Cardiol 2014;64:77-85. 
3. Hamirani YS, Wong A, Kramer CM, Salerno M. Effect of Microvascular obstruction and intramyocardial hemorrhage by CMR on LV remodeling and outcomes after myocardial infarction a systematic review and meta-analysis. JACC Cardiovasc Imaging 2014;7:940-952.

4. van Kranenburg M, Magro M, Thiele H, et al. Prognostic value of microvascular obstruction and infarct size, as measured by CMR in STEMI patients. JACC Cardiovasc Imaging 2014;7:930-939.

5. Ito H. No-reflow phenomenon and prognosis in patients with acute myocardial infarction. Nat Clin Pract Cardiovasc Med 2006;3:499-506.

6. Wong DT, Puri R, Richardson JD, Worthley MI, Worthley SG. Myocardial 'no-reflow' - Diagnosis, pathophysiology and treatment. Int J Cardiol 2013;167:1798-1806.

7. Iwakura K, Ito H, Takiuchi S, et al. Alternation in the coronary blood flow velocity pattern in patients with no reflow and reperfused acute myocardial infarction. Circulation 1996;94:1269-1275.

8. Kawamoto T, Yoshida K, Akasaka T, et al. Can coronary blood flow velocity pattern after primary percutaneous transluminal coronary angioplasty (correction of angiography) predict recovery of regional left ventricular function in patients with acute myocardial infarction? Circulation 1999; 100:339-345.

9. Shintani Y, Ito H, Iwakura K, et al. Prediction of wall motion recovery from the left anterior descending coronary artery velocity pattern recorded by transthoracic doppler echocardiography in patients with anterior wall myocardial infarction retrospective and prospective studies. Jpn Circ J 2001;65:717-722.

10. Wakatsuki T, Nakamura M, Tsunoda T, et al. Coronary flow velocity immediately after primary coronary stenting as a predictor of ventricular wall motion recovery in acute myocardial infarction. J Am Coll Cardiol 2000;35:18351841.

11. Moya Mur JL, Guzmán G, Catalán P, et al. Yield of transthoracic echocardiography with high frequency transducer in the study of the anterior descending coronary artery. Rev Esp Cardiol 2001;54:16-21.

12. Okamura A, Ito $\mathrm{H}$, Iwakura $\mathrm{K}$, et al. Usefulness of a new grading system based on coronary flow velocity pattern in predicting outcome in patients with acute myocardial infarction having percutaneous coronary intervention. Am J Cardiol 2005;96:927-932.

13. Tani T, Tanabe K, Kureha F, et al. Transthoracic Doppler echocardiographic assessment of left anterior descending coronary artery and intramyocardial artery predicts left ventricular remodeling and wall-motion recovery after acute myocardial infarction. J Am Soc Echocardiogr 2007;20:813-819.

14. Hozumi T, Kanzaki Y, Ueda Y, et al. Coronary flow velocity analysis during short term follow up after coronary reperfusion: use of transthoracic Doppler echocardiography to predict regional wall motion recovery in patients with acute myocardial infarction. Heart 2003;89:1163-1168.
15. Okcular I, Sezer M, Aslanger E, et al. The accuracy of deceleration time of diastolic coronary flow measured by transthoracic echocardiography in predicting long-term left ventricular infarct size and function after reperfused myocardial infarction. Eur J Echocardiogr 2010;11:823-828.

16. Iwakura K, Ito H, Kawano S, et al. Assessing myocardial perfusion with the transthoracic Doppler technique in patients with reperfused anterior myocardial infarction: comparison with angiographic, enzymatic and electrocardiographic indices. Eur Heart J 2004;25:1526-1533.

17. Saraste A, Koskenvuo JW, Saraste M, et al. Coronary artery flow velocity profile measured by transthoracic Doppler echocardiography predicts myocardial viability after acute myocardial infarction. Heart 2007;93:456-457.

18. Iancu A, Ober C, Bondor CI, Cadiș H. Microvascular effect of intracoronary eptifibatide in acute myocardial infarction. Cardiology 2012;123:46-53.

19. Van de Werf F, Ardissino D, Betriu A, et al; the Task Force on the Management of Acute Myocardial Infarction of the European Society of Cardiology. Management of acute myocardial infarction in patients presenting with ST-segment elevation. The Task Force on the Management of Acute Myocardial Infarction of the European Society of Cardiology. Eur Heart J 2003;24:28-66.

20. The Thrombolysis in Myocardial Infarction (TIMI) trial. Phase I findings. TIMI Study Group. N Engl J Med 1985;31:932-936.

21. van 't Hof AW, Liem A, Suryapranata H, Hoorntje JC, de Boer MJ, Zijlstra F. Angiographic assessment of myocardial reperfusion in patients treated with primary angioplasty for acute myocardial infarction: myocardial blush grade. Zwolle Myocardial Infarction Study Group. Circulation 1998;97:2302-2306.

22. Hozumi T, Yoshida K, Ogata Y, et al. Noninvasive assessment of significant left anterior descending coronary artery stenosis by coronary flow velocity reserve with transthoracic color Doppler echocardiography. Circulation 1998;97:1557-1562.

23. Zhang YL, Wei M, Han BB, Xue XP, Zhang WX, Wang M. Coronary flow velocity pattern and recovery of regional left ventricular function: the relationship observed in patients with reperfused acute myocardial infarction. Tex Heart Inst J 2010;37:166-171.

24. Cuculi F, De Maria GL, Meier P, et al. Impact of microvascular obstruction on the assessment of coronary flow reserve, index of microcirculatory resistance, and fractional flow reserve after ST-segment elevation myocardial infarction. J Am Coll Cardiol 2014;64:1894-1904.

25. Abanador-Kamper N, Karamani V, Kamper L, Brinkmann H, Haage P, Seyfarth M. Temporal course of microvascular obstruction after myocardial infarction assessed by MRI. Diagn Interv Radiol 2015;22:151-155.

26. Galiuto L, Lombardo A, Maseri A, et al. Temporal evolution and functional outcome of no reflow: sustained and spontaneously reversible patterns following successful coronary recanalisation. Heart 2003;89:731-737. 